\title{
Research on the Improved Particle Swarm Optimization Algorithm Apply- ing in the Reservoir Optimal Scheduling
}

\author{
Hu Rui-peng* \\ School of Mathematics \& Computer Science of Wuhan Polytechnic University, Wuhan, 430023, China
}

\begin{abstract}
Particle Swarm Optimization Algorithm (PSO) is often used to solve complex optimal scheduling. But in the process of particle swarm optimization, the homogenization of particle swarm is prone to premature homogenization result. In order to solve this problem, this paper proposes the new mechanisms to assign the value to inertia factor adaptively and dynamically with the evolution speed factor and mean fitness variance of population diversity factor to improve the traditional linear method. Then the improved particle swarm optimization algorithm is applied to the actual reservoir optimal scheduling to verify that the algorithm has faster homogenization speed to get the global extreme and overcomes the shortcomings of easily fall into local optimum. This provides a new way for the reservoir optimal scheduling problem.
\end{abstract}

Keywords: Adaptability, evolution speed, inertia factor, mean fitness variance of population diversity, particle Swarm optimization algorithm, reservoir optimal scheduling.

\section{INTRODUCTION}

The particle swarm optimization (PSO) is an evolutionary computing technology. Based on the research on the preying behaviors of bird flocks, Dr. Eberhart and Kennedy proposed an adaptive probability optimization technology for complex system computing to simulate the intelligent behaviors of the swarm [1] in 1995. A problem solution corresponds to the position of a bird in the search space in PSO algorithm. The birds are called "particles" or "main body". Each particle has its own position and speed (to determine flight direction and distance) as well as a fitness value determined by the optimized function. Particles memorize and track the currently optimal particles and iteratively search the solution space. Each iteration process is not fully random. If a better solution is found, the next solution will be searched based on the current solution. The PSO algorithm features simple implementation and adjustment of few parameters. Now the PSO algorithm is extensively used in function optimization, neutral network training and fuzzy system control and has achieved better effect. The PSO algorithm is extensively applied in the reservoir scheduling in China, but the PSO algorithm has problems in some complex and strongconstrained system optimization. The main problem is that the "homogenization" in the particle swarm optimization leads to premature homogenization. For the above problem, some experts and scholars have proposed some improvement method. E.g. Shi Y. [2] proposed the PSO algorithm with linear decrease of inertia factor $w$. Hong Gui-shan [3] and $\mathrm{Xu}$ Cong-dong [4] proposed PSO algorithm with adaptive adjustment inertia factor $w$. The linear decrease inertia factor

*Address correspondence to this author at the School of Mathematics \& Computer Science of Wuhan Polytechnic University, Wuhan, 430023, China; E-mail: hurpq@163.com proposed by Shi Y. is only associated with the iteration time of the algorithm and cannot truly reflect complexity of the PSO algorithm in operation. With decrease of the inertia factor, lack of global search capability of the PSO algorithm will be highlighted in the late operation period. Hong Guishan adopts a strategy to identify $w_{i}=w_{\max }-\left(w_{\max }-w_{\min }\right) t / t_{\max }(t$ is the current iteration time and $t_{\max }$ is the maximum iteration time) of $i^{\text {th }}$ iteration within the weight range of the inertia factor, so the designed inertia factor will linearly reduce $w$ value with iteration, but it does not solve the problem that too small $w$ leads to loss of the new space expansion capability. The author adopts one adaptive and dynamic inertia factor $w$ mechanism, which is associated with the evolution speed of particle swarm and mean fitness variance of population diversity in order to discuss application of the PSO algorithm in the reservoir optimal scheduling.

\section{DESCRIPTION OF PSO ALGORITHM}

The PSO algorithm is initialized as a random particle swarm, which is also called as the random solution, and then searches the optimal solution via iteration. The particle updates itself by tracking two "extremes" in each iteration. The first extreme is the optimal solution searched by a particle and is called an individual extreme $P_{i}$. Another extreme is the currently searched optimal solution in the whole population and is called the global extreme $P_{g}$. The particle neighbors can be partial particles instead of whole population, so the extreme in all neighbors will be a local extreme. After two optimal solutions are searched, the particle can update self-position and flight speed according to the following equation (1) and (2).

Assuming $m$ particles, the information on the particle $i$ can be expressed with D-dimension vector. The position is 
expressed as $X_{i}=\left(X_{i 1}, X_{i 2}, \ldots, X_{i d}\right), i=1,2, \ldots, m$. The speed is expressed as $V_{i}=\left(V_{i 1}, V_{i 2}, \ldots, V_{i d}\right) . X_{i}$ is substituted in the fitness function to get the particle fitness $f\left(X_{i}\right)$. After the individual extreme $P_{i}$ and global extreme $P_{g}$ are found, the flight speed and position of the particles can be updated according to the equation (1) and (2) $[1,2]$.

$$
\begin{aligned}
& V_{i d}=w V_{i d}+c_{1} r_{1}\left(P_{i d}-X_{i d}\right)+c_{2} r_{2}\left(P_{g d}-X_{i d}\right) \\
& X_{i d}=X_{i d}+V_{i d}
\end{aligned}
$$

In this equation, $i=1,2, \ldots, m ; d=1,2, \ldots, D . w$ is a nonnegative constant and is called as the inertia factor. The value can linearly decrease between 0.9 and 0.4 with iteration. $c_{1}$ and $c_{2}$ are called as the learning factor and generally takes the value 2. $r_{1}$ and $r_{2}$ are random numbers between 0 and 1 . $V_{i d} \in\left(-V_{\max }, V_{\max }\right), V_{\max }$ is a constant. Generally the iteration termination condition is that the optimal position searched currently by the maximum iteration time and particle swarm meets the fitness threshold.

\section{IMPROVEMENT OF PSO ALGORITHM}

The key to solve the problem that the "homogenization" leads to premature homogenization in the particle swarm optimization of the PSO algorithm is to select a reasonable value of the inertia factor $w$. The inertia factor $w$ can adjust the local and global search capability of the PSO algorithm. A bigger $w$ value can facilitate extension of the search space in the initial period of the iteration, but the local micro adjustment capability is weak. The smaller $w$ value can facilitate local refined search in the later period of iteration, but the capability of extending new space is weaker. Many scholars propose to linearly decrease value of the inertia factor $w$ in iteration, e.g. take the value somewhere between 0.4 and 0.9 [5-7]. Although this value assignment method can obviously improve accuracy of the algorithm, but this linear decrease is only associated with iteration time in iteration and cannot truly reflect complexity and non-linear change of the PSO algorithm in iteration and cannot solve the problem that the smaller $w$ value will lead to the loss of capability of extending new space. This paper, constructs the inertia factor $w$ with dynamic adaptive value based on evolution speed and population diversity of the particle swarm in order to improve the traditional particle swarm algorithm $[4,5]$.

\subsection{Evolution Speed of Particle Swarm}

Generally the value of the fitness function is always set larger than 0 in the PSO algorithm. The global extreme is not only obtained from iteration of the individual extreme $P_{i}$, but also reflects operation effect of all particles in the particle swarm. The fitness $f_{p g}(t)$ of the global extreme $P_{g}(t)$ of current iteration is always larger or equal to the fitness $f_{p g}(t-1)$ of the global extreme $P_{g}(t-1)$ of previous iteration, namely

$\Delta f^{t}=\left|f_{p g}(t)-f_{p g}(t-1)\right| \rightarrow 0$

So the evolution speed $s$ of the particle swarm can be expressed as follows:

$$
s=\left|\frac{\max \left\{f_{p g}(t), f_{p g}(t-1)\right\}-\min \left\{f_{p g}(t), f_{p g}(t-1)\right\}}{f_{1}(t)}\right|
$$

Where in, $\quad f_{1}(t)=\max \left\{\Delta f^{1}, \Delta f^{2}, \ldots, \Delta f^{t}\right\} \quad$, $\Delta f^{t}=\left|f_{p g}(t)-f_{p g}(t-1)\right|$.

The initial fitness, initial individual extreme and initial global extreme of the particle are computed according to the initial position vector and speed $s$ vector of the initial particle. All particles will continue, updating speed and position, compute fitness of the particles, and update the individual extremes and global extremes of particles in the optimization iteration of the particle swarm. With the fitness value of global extremes, the evolution speed $s$ of the particle swarm can be computed according to the equation (3). It indicates that the evolution speed $s$ of the particle swarm is determined dynamically by different fitness in iteration. The value is between 0 and 1 . A bigger value indicates quicker evolution speed. A smaller value indicates slow evolution speed.

\subsection{Mean Fitness Variance of Population Diversity}

The population diversity is also one important factor to affect performance of the PSO algorithm. Too quick loss of the populations will lead to obvious "homogenization" in the particle swarm optimization and premature homogenization of the PSO algorithm. This paper defines the mean fitness variance $\sigma^{2}$ of the particle swarm population by using the variance concept similar to the statistics to reflect diversity of the particle swarm. It is expressed as follows:

$\sigma^{2}=\frac{1}{m} \sum_{i=1}^{m}\left(\frac{f^{i}(t)-f_{\text {avg }}}{f_{2}(t)}\right)^{2}$

In this equation, $m$ indicates the size of the particle swarm. $f^{i}(t)$ indicates the fitness of $\mathrm{i}^{\text {th }}$ particles and $f_{\text {avg }}$ indicates the mean fitness of the particle swarm.

$$
\begin{aligned}
& f_{2}(t)=\max \left\{\left|f^{1}(t)-f_{\text {avg }}\right|,\left|f^{2}(t)-f_{\text {avg }}\right|, \ldots,\left|f^{m}(t)-f_{\text {avg }}\right|\right\}, \\
& \text { and }\left|f^{i}(t)-f_{\text {avg }}\right| \rightarrow f_{2}(t) .
\end{aligned}
$$

From the equation (4), the mean fitness variance $\sigma^{2}$ of the population is between 0 and 1. A smaller value indicates the higher homogenization of particles and higher homogenization of the population. A bigger value indicates the lower homogenization of particles and particles under the phase of extending new search space, so the population is diversified.

\subsection{Value of Dynamic Adaptive Inertia Factor $w$}

The function of inertia factor $w$ of the PSO algorithm is constructed according to the particle swarm evolution speed $s$ and mean fitness variance $\sigma^{2}$ of population obtained from the equation (3) and (4):

$$
w=f_{3}\left(s, \sigma^{2}\right)
$$


A bigger evolution speed $s$ of the particle swarm indicates quicker evolution of the particle swarm, so the PSO can continuously search for a long time in a bigger search space. The global search capability of the PSO algorithm is enhanced by increasing the inertia factor $w$. The evolution speed $s$ of the reverse particle swarm will gradually decrease. At this time, $w$ value should decrease to enhance the local and subtle search capability of the algorithm, so the value of the inertia factor should gradually decrease with decrease of the evolution speed $s$. On the other hand, the global search capability should be enhanced in the early period of the particle swarm optimization. The inertia factor $w$ should gradually increase with increase of the population diversity of the partial swarm. To enhance the local search capability in the late period, the inertia factor $w$ should gradually decrease with decrease of the population diversity.

In a word, the value of the inertia factor $w$ should increase with increase of the evolution speed $s$ of the particle swarm and the mean fitness variance $\sigma^{2}$ of the population and will decrease with decrease of the evolution speed $s$ of the particle swarm and the mean fitness variance $\sigma^{2}$ of the population, so the value function of the inertia factor $w$ is expressed as follows:

$$
w=f_{3}\left(s, \sigma^{2}\right)=k_{1} s+k_{2} \sigma^{2}+w_{i n i}
$$

In this equation, $w_{\mathrm{ini}}$ is the initial value of the inertia factor. $k_{1}$ and $k_{2}$ are the speed inertia factor and variance inertia factor, respectively, so the value of $s$ is between 0 and 1 , the value of $\sigma^{2}$ is between 0 and 1 , and the value of $w$ is [ $w_{\text {ini }}$, $w_{i n i}+k_{1}+k_{2}$ ). The inertia factor $w$ can be adjusted adaptively with change of the evolution speed $s$ of the particle swarm and the mean fitness variance $\sigma^{2}$ of the population in order to improve the performance of PSO algorithm.

\subsection{Homogenization of Inertia Factor $w$}

Assuming that $\mathrm{t}^{\text {th }}$ inertia factor is $w(t)$ and $(\mathrm{t}+1)^{\text {th }}$ inertia factor is $w(t+1)$, the following equation can be obtained from the equation (6):

$$
\begin{aligned}
& \Delta w=w(t+1)-w(t) \\
& =k_{1}(s(t+1)-s(t))+k_{2}\left(\sigma^{2}(t+1)-\sigma^{2}(t)\right) \\
& =k_{1} \Delta s+k_{2} \Delta \sigma^{2}
\end{aligned}
$$

The following equation can be obtained from the equation (3):

$$
\begin{aligned}
& f_{1}(t+1)=\max \left\{\Delta f^{1}, \Delta f^{2}, \ldots, \Delta f^{t}, \Delta f^{t+1}\right\} \\
& =\max \left\{f_{1}(t), \Delta f^{t+1}\right\}=f_{1}(t)
\end{aligned}
$$

The following equation can be obtained

$$
\Delta s=s(t+1)-s(t)=\frac{\Delta f^{t+1}}{f_{1}(t+1)}-\frac{\Delta f^{t}}{f_{1}(t)}=\frac{\Delta f^{t+1}-\Delta f^{t}}{f_{1}(t)}
$$

As described in the section 3.1, $\Delta f^{t}=\left|f_{p g}(t)-f_{p g}(t-1)\right| \rightarrow 0$, with $t \rightarrow \infty, \Delta s \rightarrow 0, w$ will converge for $s$.
The following equation can be obtained from the equation (4):

$$
\begin{aligned}
& \Delta \sigma^{2}=\sigma^{2}(t+1)-\sigma^{2}(t) \\
& =\frac{1}{m} \sum_{i=1}^{m}\left(\frac{f^{i}(t+1)-f_{\text {avg }}(t+1)}{f_{2}(t+1)}+\frac{f^{i}(t)-f_{\text {avg }}(t)}{f_{2}(t)}\right) * \\
& \left(\frac{f^{i}(t+1)-f_{\text {avg }}(t+1)}{f_{2}(t+1)}-\frac{f^{i}(t)-f_{\text {avg }}(t)}{f_{2}(t)}\right)
\end{aligned}
$$

As described in the section 3.3,

$\left|f^{i}(t)-f_{\text {avg }}(t)\right| \rightarrow f_{2}(t)$

With iteration time, $t \rightarrow \infty, \Delta \sigma^{2} \rightarrow 0, w$ will converge for $\sigma^{2} . \Delta s$ and $\Delta \sigma^{2}$ are the function of different independent variables, so the parameter $s$ and $\sigma^{2}$ of the inertia factor are independent of each other.

\section{APPLICATION OF IMPROVED PSO ALGORITHM IN RESERVOIR OPTIMIZED SCHEDULING}

The optimized scheduling of reservoirs is a typical nonlinear, multi-phase, constrained and dynamic combination optimization. Taking a reservoir as one example, validate the PSO algorithm. This reservoir is an annual adjustment reservoir and provides power generation and water supply as main function and irrigation and flood control as assisted function. The normal water level is $760 \mathrm{~m}$ and the corresponding capacity is 2.15 billion $\mathrm{m}^{3}$. The dead water level is $720 \mathrm{~m}$ and the corresponding capacity is 0.79 billion $\mathrm{m}^{3}$. The limit falling water level is $715 \mathrm{~m}$ and the corresponding capacity is 0.68 billion $\mathrm{m}^{3}$. The installed capacity is 0.6 million $\mathrm{kW}$ to ensure 0.18 million $\mathrm{kW}$. The limit water level for flood control is not over $755 \mathrm{~m}$ in summer (Jun-Sep). Taking the runoff into the reservoir as one example and the month as the unit, the reservoir scheduling is optimized to maximize the annual power generation under the premise of ensuring flood control, irrigation, water supply and navigation. At this time, the target function for optimized scheduling is expressed as follows [8]:

$$
F=\max \sum_{k=1}^{12} A Q_{k} H_{k} M_{k}
$$

In this equation, $A$ is the output constant of the power station, $Q_{k}$ is the power generation flow at $\mathrm{k}^{\text {th }}$ interval, $H_{k}$ is the mean water head of the reservoir at $\mathrm{k}^{\text {th }}$ interval, and $M_{k}$ is the interval duration, namely the hours at $\mathrm{k}^{\text {th }}$ interval.

The main constraints include the reservoir water volume balance, reservoir capacity, reservoir discharging water flow and power station output in optimized scheduling. Assuming that the size of the water flow particle swarm, the maximum number of iterations, the maximum moving speed of water flow particle $V_{\max }=0.5 \mathrm{~m} / \mathrm{s}$, the value of learning factor $c_{1}$ and $c_{2}$ are 2 in the algorithm validation. Generally the value of the inertia factor $w$ linearly decreases from 0.9 to 0.4 in the traditional PSO algorithm. This paper uses the adaptive and dynamic inertia factor method. The value of $w$ is $\left[w_{\text {ini }}\right.$, $w_{i n i}+k_{1}+k_{2}$ ). Assuming that the value of the inertia factor 
Table 1. Comparison of solutions of improved PSO algorithm and traditional PSO algorithm.

\begin{tabular}{|c|c|c|c|c|c|c|c|c|c|}
\hline Month & $\begin{array}{c}\text { Flow into } \\
\text { Reservoir } \\
\quad / \mathrm{m}^{3} \cdot \mathrm{s}^{-1}\end{array}$ & \multicolumn{4}{|c|}{ Results of Improved PSO Algorithm } & \multicolumn{4}{|c|}{ Results of Traditional PSO Algorithm } \\
\hline 5 & 240 & 724.7 & 197.1 & 0 & 180 & 724.5 & 198.9 & 0 & 181.3 \\
\hline 7 & 364 & 748.2 & 164.5 & 0 & 180.0 & 747.5 & 166.7 & 0 & 181.2 \\
\hline 8 & 463 & 755.0 & 426.8 & 0 & 439.4 & 755.0 & 421.9 & 0 & 428.1 \\
\hline 9 & 411 & 755.0 & 411.0 & 0 & 426.5 & 755.0 & 411.0 & 0 & 426.5 \\
\hline 1 & 117 & 758.5 & 141.9 & 0 & 180.0 & 758.0 & 143.2 & 0 & 181.0 \\
\hline 2 & 105 & 756.2 & 143.9 & 0 & 180.0 & 755.5 & 148.7 & 0 & 184.5 \\
\hline 3 & 103 & 753.6 & 146.6 & 0 & 180.0 & 752.5 & 149.8 & 0 & 182.3 \\
\hline 4 & 150 & 720.0 & 556.6 & 0 & 523.9 & 720.0 & 541.6 & 0 & 507.1 \\
\hline \multicolumn{6}{|c|}{ Annual power generation: 2.1493 billion kW.h } & \multicolumn{4}{|c|}{2.1324 billion $\mathrm{kW} . \mathrm{h}$} \\
\hline \multicolumn{6}{|c|}{ Computing time: $36 \mathrm{~s}$} & \multicolumn{4}{|c|}{$34 \mathrm{~s}$} \\
\hline
\end{tabular}

$w_{\text {ini }}=0.4$ and the speed inertia factor $k_{1}$ and the variance inertia factor $k_{2}$ meet the condition $0 \leq k_{1}+k_{2} \leq 0.5\left(k_{1}\right.$ and $k_{2}$ are more than 0$)$, the PSO algorithm is validated by taking 7 values of $\mathrm{k} 1$ and $\mathrm{k} 2\left(\left(k_{1}, k_{2}\right)=\{(0,0.5),(0.1,0.4),(0.2,0.3)\right.$, $(0.25,0.25),(0.3,0.2),(0.4,0.1),(0.5,0)\})$.

After comparison, the improved PSO algorithm has the maximum performance when $k_{1}=0.4$ and $k_{2}=0.1$.

To validate that the improved PSO algorithm is advantageous to the traditional PSO algorithm, this paper also optimizes reservoir scheduling by using a traditional PSO algorithm. Assuming that the value of inertia factor $w$ is 0.9 and the target function, constraint conditions and other parameters are not changed, the results for reservoir optimal scheduling are shown as the Table 1. It indicates that the computing time of the improved PSO algorithm is $36 \mathrm{~s}$ and is less than the time (34s) of the traditional PSO algorithm, but the difference is smaller. The annual power generation is 2.1493 billion $\mathrm{kWh}$, which is 16.9 million $\mathrm{kWh}$ more than the traditional PSO algorithm, so the improvement strategy of the PSO algorithm is effective. The adaptive value of the inertia factor $w$ can ensure adaptive adjustment of the value of $w$ according to the actual conditions in optimization of the particle swarm and extends the global search of the PSO algorithm, overcome the homogenization of the local optimization, and improves algorithm performance.

\section{CONCLUSION}

To solve the premature homogenization problem caused by "homogenization" in particle swarm optimization of the PSO algorithm, this paper introduces evolution speed $s$ of particle swarm and diversity fitness variance $\sigma^{2}$ of populations, constructs random value of the adaptive and dynamic inertia factor $w$ and discusses homogenization of the inertia factor $w$ and independence of parameter $s$ and $\sigma^{2}$ in order to improve the traditional PSO algorithm. This paper applies the improved PSO algorithm into the optimized scheduling of the reservoir. The example demonstration shows that the adaptive and dynamic adjustment of $w$ value based on actual condition can overcome the weaknesses of weaker local optimization capability and local optimization trap of the PSO algorithm and improve reliability of global optimization. The improved PSO algorithm is easy to program, occupies smaller computer resources, and offers faster processing speed and precision, so it provides new effective means for reservoir optimal scheduling.

\section{CONFLICT OF INTEREST}

The author confirms that this article content has no conflict of interest. 


\section{ACKNOWLEDGEMENTS}

The work described in this paper was jointly supported by Wuhan Polytechnic University, Shanghai University of Engineering Science, and Changjiang River Scientific Research Institute. I acknowledge my Dr. Shao Ming, Dr. Wang Gang, Dr. Wang Han-dong for giving me courage and supports.

\section{REFERENCES}

[1] R. Eberhart, and J. Kennedy, "A new optimizer using particle swarm theory," In: Proceedings of the $6^{\text {th }}$ international Symposium on Micro Machine and Human Science, 1995, Nagoya: IEEE Press, pp. 39-43,1995.

[2] Y. Shi, and R. Eberhart, "A modified particle swarm optimizer," In: Proceedings of the IEEE World Congress on Computation Intelligence, Piscataway: IEEE Press, pp. 69-73,1998.
[3] H. Gui-shan, and X. Bo, "An improved particle swarm optimization algorithm," Journal of Guangdong University of Petrochemical Technology, pp. 75-78, April 2013.

[4] X. Cong-dong, and C. Chun, "A particle swarm optimization algorithm of adaptive dynamic control parameter," Journal of Computer Engineering, vol. 2013, no. 10, pp. 203-207, Oct. 2013.

[5] K. E. Parsopoulos, and M. N. Vrahatis, "Particle swarm optimization in multiobjective problems," In: Proceedings of the ACM 2002 Symposium on Applied Computing, Amsterdam: IOS Press, pp. 603-607, 2002.

[6] V. John, E. Trucco, and S. Ivekovic, "Markerless human articulated tracking using hierarchical particle swarm optimization," Image and Vision Computing, pp. 1530-1547, Nov. 2010.

[7] S. Tong-fen, A Modified Immune Particle Swarm Algorithm and Research on Cascaded Reservoir Optimal Scheduling, Taiyuan: Taiyuan University of Technology, 2010.

[8] Z. Yong-chuan, Optimal Control of Water Resources System, Wuhan: Huazhong University of Science \& Technology Press, 1993.

(C) Hu Rui-peng; Licensee Bentham Open.

This is an open access article licensed under the terms of the Creative Commons Attribution Non-Commercial License (http://creativecommons.org/licenses/by-nc/3.0/) which permits unrestricted, non-commercial use, distribution and reproduction in any medium, provided the work is properly cited. 